\title{
Connectivity of Large-Scale WSNs in Fading Environments under Different Routing Mechanisms
}

\author{
Prodromos-Vasileios Mekikis*, Elli Kartsakli*, Aris S. Lalos*, \\ Angelos Antonopoulos*†, Luis Alonso*, and Christos Verikoukis ${ }^{\dagger}$ \\ * Signal Theory and Communications Dept., Technical University of Catalonia, Barcelona, Spain \\ ${ }^{\dagger}$ Telecommunications Technological Centre of Catalonia (CTTC), Castelldefels, Barcelona, Spain \\ e-mail:\{vmekikis, ellik, aristeidis.lalos, angelos.antonopoulos, luisg\}@
}

\begin{abstract}
As the number of nodes in wireless sensor networks (WSNs) increases, new challenges have to be faced in order to maintain their performance. A fundamental requirement of several applications is the correct transmission of the measurements to their final destinations. Thus, it is crucial to guarantee a high probability of connectivity, which characterizes the ability of every node to report to the fusion center. This network metric is strongly affected by both the fading characteristics and the different routing protocols that are used for the dissemination of data. In this paper, we study the probability of a network to be fully connected for two widely employed routing mechanisms, namely unicast and $K$-anycast. The analytical derivations and the simulations evaluate the trade-offs among the different routing mechanisms and provide useful guidelines on the design of WSNs.

Index Terms-Wireless sensor network, Connectivity, Routing, Poisson point process
\end{abstract}

\section{INTRODUCTION}

Recent Internet of Things infrastructures result in the rapid and random deployment of large-scale multihop wireless sensor networks (WSNs) in urban environments. In such networks, the ability to guarantee the delivery of all information from the source nodes to the final destination, e.g., a fusion center, is crucial for their reliability. In particular, there are many applications in different domains (such as smart grids [1], intrusion detection [2], vehicular networks [3], etc.) that demand the existence of a path between any two nodes in the network. Therefore, in contrast to small-scale WSNs, the random deployment of a large number of sensors raises important issues with regard to i) the routing mechanisms that are employed to ensure that information is delivered efficiently to the fusion center, and ii) link failures due to channel conditions in conjunction with the random deployment, that could be induced without appropriate network design.

In large-scale WSNs, there is a trade-off between energy consumption and quality of service (QoS), which significantly depends on the employed routing mechanism. In the most common routing schemes, a source node either communicates with one receiver (i.e., usually its nearest neighbor based on a routing table) with a point-to-point unicast transmission or broadcasts its message to every node in the network with a point to multipoint transmission. In the unicast case, the total energy consumption of the network is lower since only one receiver participates in each hop [4], which, however, results in lower QoS. On the other hand, in the broadcast case, more users participate in the message reception, thus resulting in higher total power consumption. Yet, the higher number of receivers increases the diversity gain, leading to QoS improvement. In an intermediate scheme, known as $K$-anycast, a source node transmits its data to a group of the $K$ nearest out of $n$ nodes [5]. The extreme cases of 2anycast and $n$-anycast (i.e., broadcast) provide the bounds of the $K$-anycast routing mechanism.

Additionally to the routing mechanism, the network reliability is compromised due to path failures that may occur either due to the random topology or the channel fading conditions. As a result, there is a need to quantify the ability of a node to report at the fusion center. This can be measured with the probability of connectivity [6], [7]. Generally, when this probability becomes one, it can be shown that there is a path between any pair of nodes. In the literature, there are many works that study the probability of connectivity in wireless networks [7]-[9]. In [7], the author examines a wireless multihop network and calculates the critical radio range that is required to achieve a minimum number of neighbors per node, so-called minimum node degree. Through the minimum node degree, the author derives the connectivity of the network. This work is extended in [8] by taking into account the boundary effects in a finite wireless network, in order to improve the accuracy of the overall network connectivity. Moreover, in [9], the authors derive the mean node degree, which provides the average number of nodes that are in the vicinity of each node. This metric provides insights about the connectivity of a network (i.e., when the mean node degree is above one, then the network starts to consist of a finite number of clusters) and the minimum delay of information propagation [10].

The aforementioned works provide a very useful overview of the connectivity in wireless networks, but they do not take into account the effect of channel fading. Although, in the absence of fading, the nearest neighbor of a given node always provides the strongest wireless link, in fading environments, due to the randomness of the channel, the strongest link may not correspond to the nearest neighbor. Hence, the network connectivity is affected and routing can play an important role in guaranteeing the delivery of a message through multihop to the fusion center.

There are some works that study the connectivity of WSNs in fading environments i.e., [11], [12], however, to the best of 


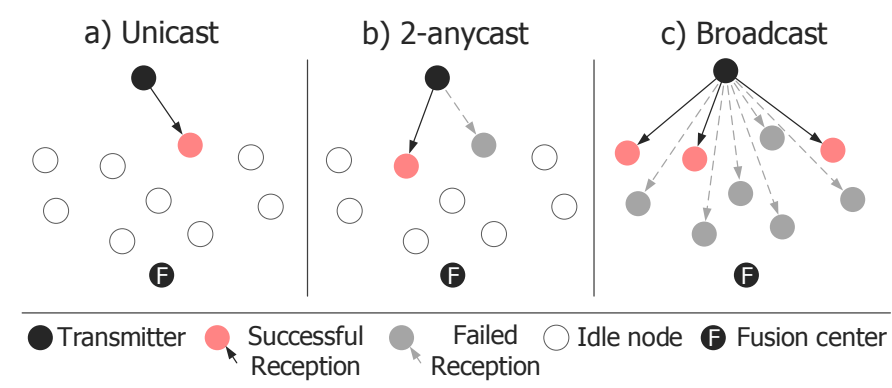

Fig. 1: The three communication scenarios a) unicast, b) 2anycast, and c) broadcast).

our knowledge, none of them considers the effects of routing in connectivity. In [11], the authors provide an empirical formula that relates connectivity with mean node degree using a lognormal shadowing radio propagation model. Nevertheless, the link probability does not take into account the density and the random locations among the nodes, which is vital for the realistic characterization of WSNs. Moreover, the authors in [12] focus on the energy savings that can be achieved by adjusting the connectivity of a network to $95 \%$, instead of having full connectivity. In their analysis, they assume lognormal shadowing model, however, they do not consider any model for the distribution of the nodes.

In this paper, we study the connectivity of a large-scale randomly deployed WSN in a fading environment for the unicast and the extreme cases of the $K$-anycast routing mechanisms (i.e., 2-anycast and broadcast). In order to model the random locations of the nodes, we distribute the nodes according to a homogeneous Poisson point process (HPPP), which is a sensible approach for modeling large-scale WSNs [13]. Our contribution is threefold: i) We analytically derive the probability of connectivity in fading environments for the unicast and the 2-anycast routing mechanisms, ii) we analytically derive the mean node degree of the network to provide insights about the broadcast scheme, and iii) we compare the two different routing mechanisms (unicast, $K$ anycast for $K=\{2, n\}$ ) and discuss the trade-offs and benefits for different kind of applications.

The remaining part of this paper is organized as follows. In Section II, we describe the system model. The mathematical derivations of the connectivity and mean node degree in the presence of fading are presented in III. The theoretical and simulation results are provided and discussed in Section IV. Finally, Section V concludes the paper.

\section{SySTEM MODEL}

We consider a large-scale wireless network consisting of a set of $n$ source nodes $S$ that transmit their messages via multi-hop communication to a fusion center $F$ that is randomly deployed in the same plane. All nodes are assumed to be on the Euclidean plane and they are represented by an independent HPPP $\Phi_{S}=\left\{x_{1}, x_{2}, \ldots, x_{n}\right\}$, where $x_{i}, \forall i \in \mathbb{N}$, denotes the location (i.e., Cartesian coordinates) of the $i^{\text {th }}$ node. $\Phi_{S}$ has an intensity $\lambda$, which corresponds to the average number of points per area unit.
In our analysis, we examine the ability of a source to connect to a given node, based on their relative distance. In a HPPP, the probability density function (PDF) of the distance $r$ of a node to its $n$th nearest neighbor is given by [13]

$$
f_{n}(r)=\frac{2}{\Gamma(n)}(\lambda \pi)^{n} r^{2 n-1} e^{-\lambda \pi r^{2}},
$$

where $\Gamma$ denotes the Gamma function given by $\Gamma(t)=\int_{0}^{\infty} x^{t-1} e^{-x} \mathrm{~d} x$. The average received power at a node is denoted by $P_{r}=P_{t} h r_{n}^{-\alpha}$, where $r_{n}$ is the distance between the receiver and its $n$th nearest neighbor (i.e., without loss of generality, we assume that the respective receiving node is located at the origin according to Slyvnyak's theorem [13]), $\alpha$ is the path loss exponent, $P_{t}$ is the transmit power of the source nodes and $h$ is the power fast fading coefficient, which is independent and identically distributed (i.i.d.). The Rayleigh fading environment is considered suitable for modeling fast fading in dense urban environments [14]. For this reason, the amplitude fading $\sqrt{h}$ is Rayleigh distributed with a scale parameter $\sigma=1$, thus $h$ is exponentially distributed with mean value $\mu$. Therefore, a node is considered connected with its $n$th nearest neighbor (i.e., is able to decode a received message), when the received signal to noise ratio (SNR) is higher than a threshold $T$, as it is given in

$$
\mathrm{SNR}_{n}=\frac{P_{t} \cdot h \cdot r_{n}^{-\alpha}}{W} \geq T,
$$

where $r_{n}$ is the Euclidean distance between the two nodes and $W$ denotes an additive white Gaussian noise power, modeled as a constant zero mean Gaussian random variable (RV).

Regarding the communication, illustrated in Fig. 1, we study two routing mechanisms separated into three scenarios. In the first scenario, shown in Fig. 1(a), each node communicates with its nearest neighbor. This is the unicast routing mechanism, in which a node is considered connected only if the nearest neighbor can decode successfully the transmitted message. The other two scenarios focus on the extreme cases of $K$-anycast. In particular, in Fig. 1(b), we illustrate the $K$-anycast scheme for $K=2$. In this scenario, each node communicates with its two nearest neighbors and the source node is considered connected if at least one of the 2 nodes is able to decode the received message. The third scenario, depicted in Fig. 1(c), is the extreme $K$-anycast case for $K=n$ (i.e., broadcast). In this case, a source node broadcasts its message to every node and it is considered connected if at least one of the receivers is able to decode the message, regardless of its proximity to the source node.

\section{NETWORK CONNECTIVITY FOR UNICAST AND $K$-ANYCAST ROUTING}

In this section, we present the mathematical derivations of the probability of connectivity for different routing mechanisms. We derive i) an exact solution for the unicast routing mechanism, ii) an approximation of the extreme case of the $K$-anycast for $K=2$, and iii) we provide insights on the broadcast case (i.e., extreme case of $K$-anycast for $K=n$ ) 
by deriving the mean node degree, which is useful for identifying the minimum transmit power with which the probability of connectivity is above zero. All metrics are essential for describing the performance of large-scale networks.

According to [15], if the number of nodes $n$ is high enough, then the following expression holds:

$$
P_{\text {con }}=P\left(d_{\min } \geq 1\right),
$$

where $d_{\min }$ denotes the minimum node degree which is the number of connections of the node with the fewest connections.

In order to determine if the minimum node degree of the network is equal or higher than one, we need to know if all nodes are connected with at least one of their neighbors. Therefore, the probability that all nodes are connected to at least one neighbor, assuming that there is statistical independence in the wireless links, is

$$
P\left(d_{\text {min }} \geq 1\right)=P\left(\mathrm{SNR}_{s} \geq T\right)^{n},
$$

where $\mathrm{SNR}_{s}$ is the signal to noise ratio at the receiving node that offers the strongest link.

In the absence of fading, due to the fact that the strongest link is always offered by the nearest neighbor, it is sufficient to identify whether the source node is able to connect with its nearest neighbor. Following a similar approach as in [7], it can be easily derived that the probability of connectivity in the absence of fading is given by

$$
P_{\text {con }}=\left(1-e^{-\lambda \pi\left(\frac{P_{t}}{T W}\right)^{2 / \alpha}}\right)^{n} .
$$

However, in our system model, all wireless links are subject to fast fading. As a result, the nearest node has not necessarily the strongest link due to the randomness that is introduced at the received power from fading. Therefore, it is possible that nodes located farther from the source node are able to decode its messages, even though the nearest node may fail.

Hence, in this case, it is important to define the routing mechanism that is used in the network, before proceeding to the derivations of connectivity. Therefore, in the following, we study the unicast and $K$-anycast for $K=\{2, n\}$ routing mechanisms, as discussed in Section II.

\section{A. Unicast}

In the unicast case, a node is considered connected only if its nearest neighbor can decode successfully the transmitted message. Therefore, a network is considered fully connected if all $n$ nodes are connected to their nearest neighbors. Thus, the probability of connectivity is given by

$$
P_{\text {con }}=P\left(\mathrm{SNR}_{1} \geq T\right)^{n} .
$$

Therefore, we have to derive the probability that the SNR of a receiving node from its nearest neighbor is higher than a threshold $T$

$$
P\left(\mathrm{SNR}_{1} \geq T\right)=P\left(\frac{P_{t} h_{1} r_{1}^{-\alpha}}{W} \geq T\right)
$$

where $h_{1}$ is the exponentially distributed $\mathrm{RV}$ for the Rayleigh fading. In order to calculate this probability for every possible value of $r_{1}$, we need to employ the expected value of $r_{1}$, as follows:

$$
\begin{aligned}
& P\left(\mathrm{SNR}_{1} \geq T\right)=\mathbb{E}_{r_{1}}\left[P\left(h_{1} \geq \frac{T W r^{\alpha}}{P_{t}} \mid r=r_{1}\right)\right]= \\
& =\int_{0}^{\infty} P\left(h_{1} \geq \frac{T W r^{\alpha}}{P_{t}}\right) f_{1}(r) d r= \\
& =\int_{0}^{\infty} e^{\frac{-\mu T W r^{\alpha}}{P_{t}}} 2 \lambda \pi r e^{-\lambda \pi r^{2}} d r,
\end{aligned}
$$

where (8b) follows from integrating over all possible values of $r$ and (8c) follows from an exponentially distributed $\mathrm{RV}$ and from (1) for $n=1$. To solve the integral in (8c), we employ the modified Gauss-Hermite quadrature $[16]^{1}$, given by

$$
\int_{0}^{\infty} e^{-x^{2}} g(x) \mathrm{d} x=\sum_{i=1}^{q} w_{i} g\left(x_{i}\right)
$$

where $x_{i}$ are the roots and $w_{i}$ the weights of the quadrature given in [16, Table II]. The accuracy of the results is set by the degree $q$ of the quadrature.

Therefore, by (6), (8c) and (9), the probability of connectivity for the unicast routing mechanism is

$$
P_{\text {con }}=\left(\sum_{i=1}^{q} 2 w_{i} x_{i} e^{\frac{-\mu T W x_{i}^{\alpha}}{P_{t}(\lambda \pi)^{\alpha / 2}}}\right)^{n} \text {. }
$$

\section{B. 2-anycast}

The 2-anycast case is an extreme case of the $K$-anycast routing mechanism. In order to derive the probability of connectivity for this model, we have to follow a slightly different approach i.e., to study if any of the two nearest nodes is able to connect with the source node under study.

In the previous analysis, presented in Section III-A, if all nodes have at least one connection with another node, the network is connected. However, in the 2-anycast case, it is required for every node to be connected with at least one out of its two nearest nodes. Following [15], this can be expressed as

$$
P_{\text {con }}=\left(1-P_{\text {isol }}\right)^{n}=\left(1-P\left(\max \left(\mathrm{SNR}_{1}, \mathrm{SNR}_{2}\right) \leq T\right)\right)^{n},
$$

where $P_{i s o l}$ is the probability that a node is isolated and $P\left(\max \left(\mathrm{SNR}_{1}, \mathrm{SNR}_{2}\right) \leq T\right)$ is the probability of isolation from the strongest link between the two nearest nodes, which

\footnotetext{
${ }^{1}$ In fact, there is a closed form solution for (8c) given by $\int_{0}^{\infty} e^{-a x^{2}} e^{-b x} \mathrm{~d} x=\sqrt{\frac{\pi}{b}} \exp \left(\frac{a^{2}}{4 b}\right) Q\left(\frac{a}{\sqrt{2 b}}\right)$. However, it results in indeterminate values for the extreme cases, due to the multiplication of a very large number (i.e., Q-function) with a very small (i.e., exponential function). Thus, we prefer the numerical solution given by the modified Gauss-Hermite quadrature, which provides an accurate result for any case.
} 
can be written as

$$
\begin{aligned}
& P\left(\max \left(\mathrm{SNR}_{1}, \mathrm{SNR}_{2}\right) \leq T\right)= \\
& =P\left(\mathrm{SNR}_{1} \leq T, \mathrm{SNR}_{2} \leq T\right)= \\
& =P\left(h_{1} \leq \frac{T W r_{1}^{\alpha}}{P_{t}}, h_{2} \leq \frac{T W r_{2}^{\alpha}}{P_{t}}\right)= \\
& =\mathbb{E}_{r_{1}, r_{2}}\left[P\left(h_{1} \leq \frac{T W x^{\alpha}}{P_{t}}, h_{2} \leq \frac{T W y^{\alpha}}{P_{t}} \mid x=r_{1}, y=r_{2}\right)\right],
\end{aligned}
$$

where $r_{1}$ and $r_{2}$ denote the distance to the nearest and second nearest neighbor, respectively. Due to the dependence between $r_{1}$ and $r_{2}$, we will employ Jensen's inequality which provides a lower bound of (12a). Consequently, (12d) can be written as

$$
\begin{aligned}
& \mathbb{E}_{r_{1}, r_{2}}\left[P\left(h_{1} \leq \frac{T W x^{\alpha}}{P_{t}}, h_{2} \leq \frac{T W y^{\alpha}}{P_{t}} \mid x=r_{1}, y=r_{2}\right)\right] \geq \\
& \geq P\left(h_{1} \leq \frac{T W \mathbb{E}_{r_{1}}[x]^{\alpha}}{P_{t}}, h_{2} \leq \frac{T W \mathbb{E}_{r_{2}}[y]^{\alpha}}{P_{t}}\right)= \\
& =P\left(h_{1} \leq \frac{T W \mathbb{E}_{r_{1}}[x]^{\alpha}}{P_{t}}\right) P\left(h_{2} \leq \frac{T W \mathbb{E}_{r_{2}}[y]^{\alpha}}{P_{t}}\right),
\end{aligned}
$$

where (13b) follows by applying Jensen's inequality to (13a) (i.e., it can be easily proven that (13a) is exponential, thus convex) and (13c) by the independence between the RVs of (13b).

Hence, we can proceed to calculate the probabilities given in $(13 \mathrm{c})$. The mean value of the distance to the $n$th nearest node is given by

$$
\begin{aligned}
& \mathbb{E}_{r_{n}}[r]=\int_{0}^{\infty} f_{n}(r) r \mathrm{~d} r= \\
& =\int_{0}^{\infty} \frac{2}{\Gamma(n)}(\lambda \pi)^{n} r^{2 n-1} e^{-\lambda \pi r^{2}} r \mathrm{~d} r= \\
& =\frac{(2 n) !}{4^{n} n !(n-1) !} \cdot \frac{1}{\sqrt{\lambda}},
\end{aligned}
$$

where (14b) follows using (1).

To derive the probabilities given in (12a), we follow the same procedure as in (6)-(8a). Thus, (13a) can be written as

$$
\begin{aligned}
& P\left(\max \left(\mathrm{SNR}_{1}, \mathrm{SNR}_{2}\right) \leq T\right) \geq \\
& \geq\left(1-P\left(h_{1} \geq \frac{T \mathbb{E}_{r_{1}}[x]^{\alpha}}{P_{t} W^{-1}}\right)\right)\left(1-P\left(h_{2} \geq \frac{T \mathbb{E}_{r_{2}}[y]^{\alpha}}{P_{t} W^{-1}}\right)\right) \\
& =\left(1-e^{\frac{-\mu T W}{P_{t}(2 \sqrt{\lambda})^{\alpha}}}\right)\left(1-e^{\frac{-\mu T W 3^{\alpha}}{P_{t}(4 \sqrt{\lambda})^{\alpha}}}\right)
\end{aligned}
$$

where (15c) follows from the exponential distributed RV and by applying (14c) into (15b). Therefore, according to (11), the probability of connectivity for the 2-anycast scheme is given by

$$
P_{\text {con }}=\left(1-\left(1-e^{\frac{-\mu T W}{P_{t}(2 \sqrt{\lambda})^{\alpha}}}\right)\left(1-e^{\frac{-\mu T W 3^{\alpha}}{P_{t}(4 \sqrt{\lambda})^{\alpha}}}\right)\right)^{n} .
$$

\section{Broadcast}

The broadcast case is an extreme case of the $K$-anycast routing mechanism for $K=n$. In order to derive the probability of connectivity for this scheme, a similar approach to Section III-B could be applied. However, due to the complicated analysis that is attributed to the correlation on the distances among the nearest nodes, we will provide insights on this case, using the mean node degree. The mean node degree $d_{\text {mean }}$ represents the average number of nodes with which a source node can successfully exchange messages. Using the definition of the intensity measure [13], the expected number of nodes $\mathbb{E}[N(B)]$ denotes the average number of nodes that fall in an area $B$ and it is given by

$$
\mathbb{E}[N(B)]=\int_{B} \lambda(x) \mathrm{d} x=d_{\text {mean }} .
$$

In order to ensure if a source node is connected with any node, we have to confirm that the SNR at any receiving node is higher than a threshold. Hence, the mean node degree can be written as

$$
\begin{aligned}
& d_{\text {mean }}=\int_{0}^{\infty} \int_{0}^{2 \pi} P\left(\mathrm{SNR}_{x}>T\right) \lambda r \mathrm{~d} \theta \mathrm{d} r= \\
& =2 \pi \lambda \int_{0}^{\infty} P\left(h_{x}>\frac{T W r^{\alpha}}{P_{t}}\right) r \mathrm{~d} r= \\
& =2 \pi \lambda \int_{0}^{\infty} e^{-\frac{\mu T W r^{\alpha}}{P_{t}}} r \mathrm{~d} r= \\
& =\frac{2 \pi \lambda}{\alpha}\left(\frac{P_{t}}{\mu T W}\right)^{2 / \alpha} \Gamma\left(\frac{2}{\alpha}\right),
\end{aligned}
$$

where in (18a) the integral is given in polar coordinates, with $\mathrm{SNR}_{x}$ we denote the SNR of a random receiver $x$ and (18c) follows from an exponentially distributed RV.

When $d_{\text {mean }}$ reaches the value of 1 , each node of the network has on average one neighbor in its range and the connectivity probability starts to increase, as it will be also verified by the simulations. Therefore, by taking advantage of this metric, we can identify the minimum transmit power for which the probability of connectivity for the broadcast case is above zero, given by

$$
P_{t, \min }=\mu T W\left(\frac{\alpha}{2 \pi \lambda \Gamma\left(\frac{2}{\alpha}\right)}\right)^{2 / \alpha} .
$$

\section{AnAlytical and Simulation Results}

In this section, we validate the results of the mathematical analysis via extensive simulations and provide results regarding the connectivity and the mean node degree of large-scale randomly deployed WSNs. In the following, we present the setup of the simulation and the results of our experiments.

\section{A. Simulation Setup}

We study the connectivity for two routing mechanisms (i.e., unicast, $K$-anycast for $K=\{2, n\})$. In all the experiments, the channel fading gain is set at $\mu=1$, unless otherwise stated, while the path loss exponent is set at $\alpha=4$, which is a typical value for dense urban environments. The noise $W$ 
TABLE I:

SimUlATION PARAMETERS

\begin{tabular}{lll}
\hline Simulation Parameter & Symbol & Value \\
\hline \hline Path loss exponent & $\alpha$ & 4 \\
Threshold ratio & $T$ & $-10 \mathrm{~dB}$ \\
Power fading coef. mean & $\mu$ & 1 \\
Noise power & $W$ & $-70 \mathrm{dBm}$ \\
Intensity & $\lambda$ & {$\left[5 \cdot 10^{-5}, 50 \cdot 10^{-5}\right] \mathrm{per} \mathrm{m}^{2}$} \\
Area & $A$ & $1 \mathrm{~km}^{2}$ \\
\hline
\end{tabular}

is fixed at $-70 \mathrm{dBm}$ and the decoding threshold $T$ is set at $-10 \mathrm{~dB}$. Moreover, the intensity of the PPP varies between $\lambda=5 \cdot 10^{-5}$ nodes per $\mathrm{m}^{2}$ and $\lambda=15 \cdot 10^{-5}$ nodes per $\mathrm{m}^{2}$ and the simulation area is set at $A=1 \mathrm{~km}^{2}$. Hence, the number of nodes placed in the simulation area for the different simulations, varies between $n=\lambda A=50$ and $n=500$. The system parameters are summarized in Table I.

\section{B. Results}

In order to validate the derivations of Section III, we illustrate in Fig. 2 the connectivity for the unicast and 2anycast routing mechanisms in fading environments, as well as, for comparison reasons, the theoretical and simulation results of connectivity in the absence of fading. It can be seen that a close match is achieved between theory and simulations for a varying number of nodes in all cases. More specifically, in Fig. 2(a), we can see that in the absence of fading, as the number of nodes increases, the network achieves full connectivity for lower transmit power levels. Also, in Fig. 2(b), we present the impact of fading to the unicast scheme. In this case, a higher transmit power is needed in order to achieve the same level of connectivity as in Fig. 2(a). Furthermore, we can see that the number of nodes has a less important role in the presence of fading. This stems from the fact that, in the absence of fading, as the node intensity increases, the distance to the nearest neighbor (i.e., which is the strongest link in the absence of fading) decreases. Nevertheless, connectivity is less affected by the intensity in fading environments, due to the randomness in the strength of the nearest neighbor link.

Similarly, in Fig. 2(c), we illustrate the connectivity of the 2anycast scheme. As we can see, the result of the mathematical analysis follows the simulations, even though we use an approximation for the calculation of the distance to the nearest neighbors. In this case, the performance is improved (i.e., connectivity is achieved for a lower $P_{t}$ ), because the second nearest node may contribute to the connectivity of the source node when the nearest node fails. Therefore, the difference in performance between Fig. 2(b) and Fig. 2(c), shows the effects in the network connectivity of taking into account the second nearest node in fading environments.

In Fig. 3, we validate the derivation of the mean node degree and show an interesting result that indicates its importance in the broadcast case. In this double y-axis figure, the leftmost axis (green) corresponds to the mean node degree, while the rightmost axis (blue) corresponds to the probability of connectivity. As it can be seen, when the mean node degree

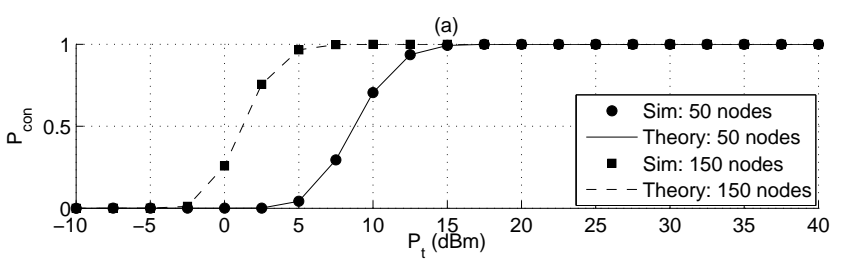

(b)
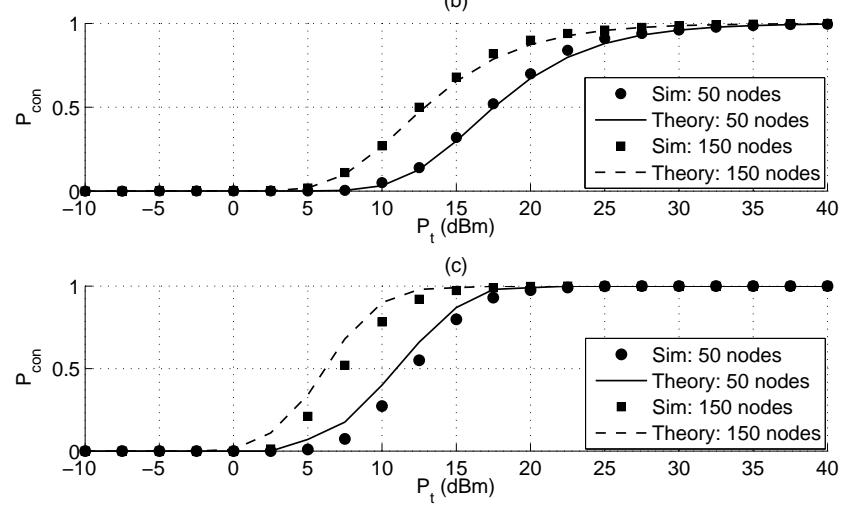

Fig. 2: Validation of the mathematical analysis for $n=50$ and $n=150$. a) Connectivity in the absence of fading, b) Unicast case, c) 2-anycast case.

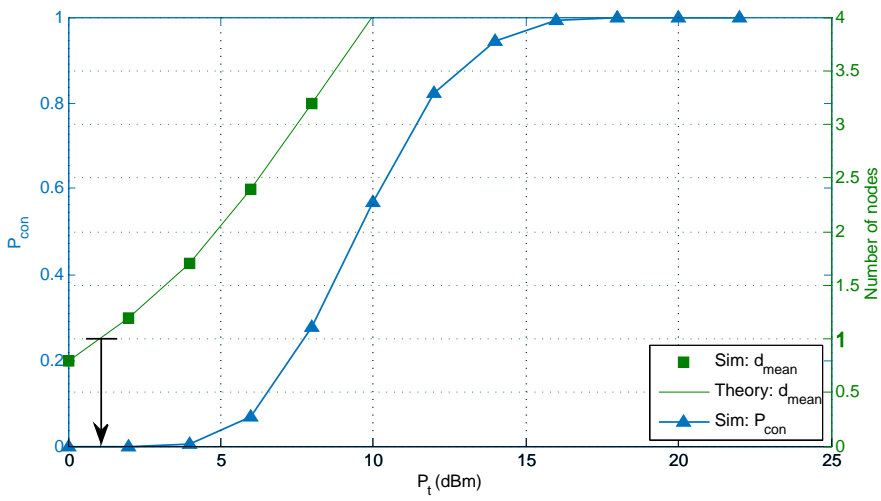

Fig. 3: Mean node degree determines when the connectivity probability is above zero in the broadcast case.

becomes equal to one, i.e., when on average every node has one neighbor, the curve of $P_{\text {con }}$ starts to increase. Therefore, it is possible to detect the transmit power that is required for the network before the connectivity is lost, which provides useful insights about connectivity in the broadcast case.

To continue with, in Fig. 4, we compare the two routing mechanisms with fading for a varying number of nodes, in order to see in more detail the performance of connectivity for each case. As it was expected, $P_{c o n}$ is higher for lower values of $P_{t}$ as the number of nodes increases from $n=50$ to $n=150$. Moreover, we show the two extreme cases of the $K$-anycast model, which is when $K=2$ (i.e., 2 -anycast) and $K=n$ (i.e., broadcast). At this point, we should point out that the curves of $P_{c o n}$ for all the intermediate cases of $K$ (i.e., from 3 to $(n-1)$ ), would be plotted in between the extreme cases. Furthermore, as the number of nodes increases, the distance between the two extremes also increases. This stems from the fact that it is more probable for the nearest node to be also the one with the strongest link for a sparse network. On 

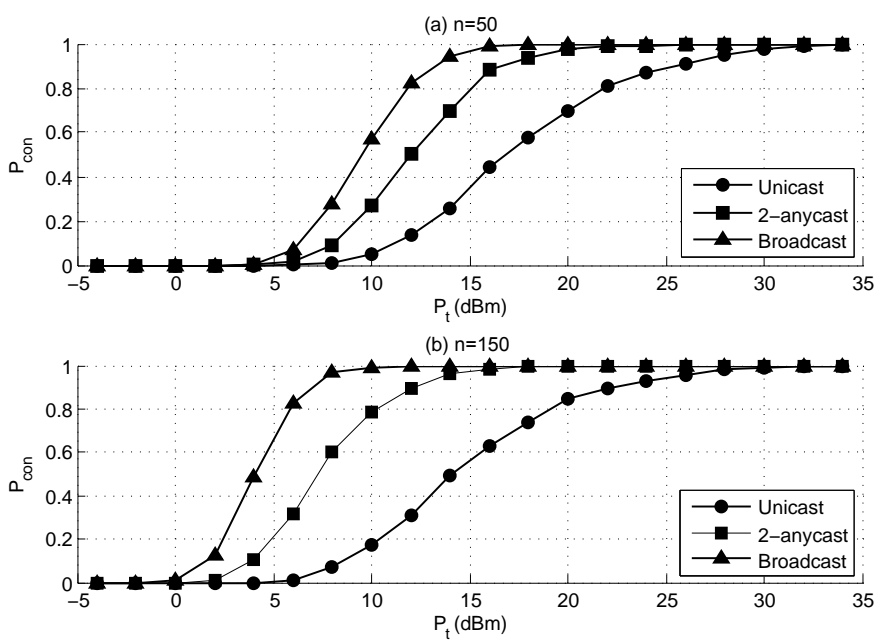

Fig. 4: Comparison of the two routing mechanisms with fading (i.e., unicast, $K$-anycast for $K=\{2, n\}$ ) for a) $n=50$, and b) $n=150$.

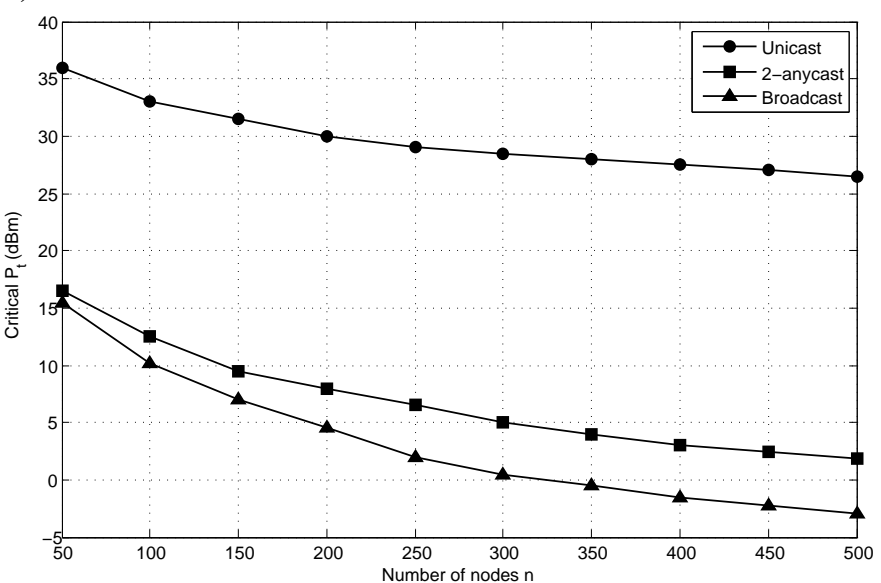

Fig. 5: Critical transmit power to achieve connectivity for the unicast and the $K$-anycast for $K=\{2, n\}$.

the other hand, as the network becomes denser, more nodes are within close proximity to a given node, therefore the likelihood that the nearest neighbor has the strongest link decreases.

Finally, in large-scale randomly deployed WSNs, it is important to know the critical $P_{t}$ for which the network remains fully connected. Therefore, in Fig. 5, we present the critical $P_{t}$ versus the number of nodes in the WSN for the two routing mechanisms. As it is illustrated, for the unicast model, if 200 nodes transmit at $30 \mathrm{dBm}$ or more, the WSN will be fully connected. On the other hand, in the 2-anycast model, substantially less power is needed (i.e., $\sim 8 \mathrm{dBm}$ ) to achieve the same probability of connectivity for the same number of nodes. Interestingly, in the case of the broadcast model, where a node is considered connected if it is connected with any of the $n$ nodes, we do not see a significant difference from the 2 -anycast case. This is reasonable since, in most cases, the strongest links are offered by the nearest neighbors. However, as expected, the performance for the broadcast case is better and a network with $n=200$ is considered fully connected if the nodes transmit their messages with approximately $5 \mathrm{dBm}$.

\section{CONClusion}

In this paper, we studied the connectivity of a large-scale randomly deployed WSN under different routing mechanisms (i.e., unicast, $K$-anycast for $K=\{2, n\}$ ). We analytically derived two important metrics for end-to-end communications, i.e., the probability of connectivity and the mean node degree and validated them through extensive Monte Carlo simulations. Moreover, we compared the different routing mechanisms and showed the circumstances under which a large-scale network is connected. Finally, we illustrated with simulations the significance of knowing the mean node degree of a network by showing its effect on the broadcast routing mechanism. As future work, we plan to continue further the analysis of connectivity in such networks by deriving the mathematical analysis for the broadcast case and also study the energy efficiency of the aforementioned routing mechanisms.

\section{ACKNOWLEDGEMENTS}

This work has been funded by AGAUR (2014-SGR$1551)$ and the research projects WSN4QoL (PIAP-GA-2011286047), ESEE (324284) and COPCAMS (332913).

\section{REFERENCES}

[1] P.-Y. Chen and S.-M. Cheng, "Smart Attacks in Smart Grid Communication Networks," IEEE Commun. Mag., vol. 50, no. 8, pp. 24-29, Aug. 2012.

[2] L. Liu, X. Zhang and H. Ma, "Percolation Theory-Based Exposure-Path Prevention for Wireless Sensor Networks Coverage in Internet of Things," IEEE Sensors Journal, vol.13, no.10, pp. 3625-3636, Oct. 2013.

[3] L. Cheng and S. Panichpapiboon, "Effects of Intervehicle Spacing Distributions on Connectivity of VANET," IEEE Commun. Mag., vol. 50, no. 10, pp. 90-97, Oct. 2012.

[4] P. Spachos, P. Chatzimisios and D. Hatzinakos"Energy Efficient Cognitive Unicast Routing for Wireless Sensor Networks," in Proc. IEEE VTC Spring 2013, pp. 1-5, Jun. 2013.

[5] X. Wang et al., "GKAR: A Novel Geographic K-anycast Routing for Wireless Sensor Networks," IEEE Trans. Parallel Distributed Systems, vol. 24, no. 5, pp. 916-925, May 2013.

[6] T.K. Philips et al., "Connectivity properties of a packet radio network model," IEEE Trans. Inform. Theory, vol. 35, no. 5, pp.1044-1047, Sep 1989.

[7] C. Bettstetter, "On the Minimum Node Degree and Connectivity of a Wireless Multihop Network," in Proc. Mobihoc 2002, pp. 80-91, Jun. 2002.

[8] Z. Khalid et al., "A Tractable Framework for Exact Probability of Node Isolation and Minimum Node Degree Distribution in Finite Multihop Networks," IEEE Trans. Veh. Technol., vol. 63, no. 6, pp. 2836-2847, Jul. 2014.

[9] W.-C. Ao and K.-C. Chen, "Cognitive Radio-Enabled Network-Based Cooperation: From a Connectivity Perspective," IEEE J. Sel. Areas Commun., vol. 30, no. 10, pp. 1969-1982, Nov. 2012.

[10] F. Baccelli et al., "Optimal paths on the space-time SINR random graph," Adv. Appl. Prob., vol. 43, no. 1, pp. 131-150, 2011.

[11] R. Hekmat and P. Van Mieghem, "Connectivity in Wireless Ad-hoc Networks with a Log-normal Radio Model," Mobile networks and applications, vol. 11, pp. 351-360, 2006

[12] T. Xiaoyuan et al., "On the Giant Component of Wireless Multihop Networks in the Presence of Shadowing," IEEE Trans. Veh. Technol., vol. 58, no. 9, pp. 5152-5163, Nov. 2009.

[13] M. Haenggi, Stochastic Geometry for Wireless Networks, Cambridge University Press, 1st ed., 2013.

[14] B. Sklar, "Rayleigh Fading Channels in Mobile Digital Communication Systems," IEEE Commun. Mag., vol. 35, no. 7, pp. 90-100, Jul. 1997.

[15] M.D. Penrose, “On $k$-connectivity for a geometric random graph,” Wiley Random Structures and Algorithms, vol. 15, no. 2, pp. 145-164, 1999.

[16] N.M. Steen et al., "Gaussian Quadratures for the integral $\int_{0}^{\infty} \mathrm{e}^{-x^{2}} f(x) \mathrm{d} x$," J. Math. Comp., no. 23, pp. 661-667, 1969. 